\title{
Gestational Age-Specific Reference Ranges for the sFIt-1/PIGF Immunoassay Ratio in Twin Pregnancies
}

\author{
Maria De La Calle ${ }^{a} \quad J u a n ~ L . D^{2}$ gado ${ }^{b}$ Stefan Verlohren ${ }^{c}$ Ana Isabel Escudero ${ }^{d}$ \\ Jose L. Bartha ${ }^{a}$ Jose M. Campillos ${ }^{e}$ Angel Aguarón De La Cruz ${ }^{f}$ \\ Frederic Chantraine ${ }^{g}$ José Ángel García Hernández ${ }^{\text {h }}$ Ignacio Herraiz ${ }^{i}$ \\ Elisa Llurba ${ }^{j, k, l}$ Hedwig Kurka $^{m}$ Ge Guo $^{n}$ Johanna Sillman ${ }^{\circ}$ Martin Hund ${ }^{\circ}$ \\ Alfredo Perales Marín ${ }^{p}$ \\ ${ }^{a}$ Division of Obstetrics and Maternal and Fetal Medicine, Hospital Universitario La Paz, Madrid, Spain; ${ }^{\mathrm{b}}$ Department \\ of Gynecology and Obstetrics, Universidad de Murcia and IMIB-Arrixaca, Murcia, Spain; 'Department of Obstetrics, \\ Charité - Universitätsmedizin, Berlin, Germany; ${ }^{d}$ Department of Obstetrics and Gynaecology, Hospital Universitario \\ Central de Asturias, Oviedo, Spain; 'Department of Obstetrics, Hospital Universitario Miguel Servet, Zaragoza, \\ Spain; fDepartment of Gynecology and Obstetrics, Hospital General Universitario Gregorio Marañón, Madrid, Spain; \\ 9Department of Obstetrics and Gynecology, Centre Hospitalier Universitaire de Liège, site CHR de la Citadelle, \\ Liège, Belgium; hepartment of Obstetrics and Gynecology, Hospital Universitario Materno Infantil de Canarias, \\ Gran Canaria, Spain; 'Department of Obstetrics and Gynaecology, Fetal Medicine Unit-SAMID, Hospital Universitario \\ 12 de Octubre, Instituto de Investigación Hospital 12 de Octubre (imas12), Universidad Complutense de Madrid, \\ Madrid, Spain; 'Obstetrics and Gynaecology Department, High Risk Unit \& Biomedical Research Institute Sant \\ Pau (IIB-Sant Pau), Sant Pau University Hospital, Barcelona, Spain; ${ }^{\mathrm{k} S c h o o l ~ o f ~ M e d i c i n e, ~ U n i v e r s i t a t ~ A u t o ̀ n o m a ~ d e ~}$ \\ Barcelona, Barcelona, Spain; 'Institute of Health Carlos III, Maternal and Child Health and Development Network \\ II (SAMID II) RD12/0026, Madrid, Spain; mBiostatistics and Data Management CPS, Roche Diagnostics GmbH,

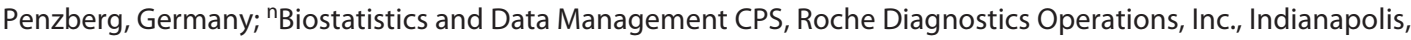 \\ IN, USA; ${ }^{\circ}$ Centralised and Point of Care Solutions, Roche Diagnostics International Ltd., Rotkreuz, Switzerland; \\ PDepartment of Obstetrics and Gynecology, Hospital Universitario y Politécnico La Fe, Valencia, Spain
}

\section{Keywords}

sFlt-1/PIGF ratio - Soluble fms-like tyrosine kinase-1 . Placental growth factor · Preeclampsia · Twin pregnancies . Reference ranges $\cdot$ Prediction · Biomarker

\section{Abstract \\ Objective: Establish reference ranges for the Elecsys ${ }^{\circledR}$ solu- ble fms-like tyrosine kinase-1 (sFlt-1)/placental growth fac- tor (PIGF) immunoassay ratio in twin pregnancies. Methods:}

Data analyzed were from 3 prospective studies: Prediction of Short-Term Outcome in Pregnant Women with Suspected Preeclampsia (PE) (PROGNOSIS), Study of Early-onset PE in Spain (STEPS), and a multicenter case-control study. Median, 5th, and 95th percentiles for sFlt-1, PIGF, and the sFIt-1/PIGF ratios were determined for normal twin pregnancies for 7 gestational windows and compared with the previous data for singleton pregnancies. Results: The reference range analysis included 269 women with normal twin pregnancies. Before 29 weeks' gestation, median, 5th, and 95th percen-
(C) 2021 The Author(s)

Published by S. Karger AG, Basel

This is an Open Access article licensed under the Creative Commons Attribution-NonCommercial-4.0 International License (CC BY-NC) (http://www.karger.com/Services/OpenAccessLicense), applicable to the online version of the article only. Usage and distribution for commercial purposes requires written permission.
Alfredo Perales Marín

Department of Obstetrics and Gynecology, Hospital Universitario y Politécnico La Fe Avda F. Abril Martorell no. 106 Torre F, 3a Planta

ES-46026 Valencia (Spain)

perales_alf@gva.es 
tiles for sFlt-1/PIGF ratios did not differ between twin and singleton pregnancies. From 29 weeks' gestation to delivery, median, 5th, and 95th percentiles for sFlt-1/PIGF ratios were substantially higher in twin versus singleton pregnancies. sFlt-1 values were higher in women with twin pregnancies across all gestational windows. PIGF values were similar or higher in twin versus singleton pregnancies; PIGF concentrations increased from 10 weeks +0 days to 28 weeks +6 days' gestation. Conclusions: Reference ranges for the sFlt-1/PIGF ratio are similar in women with twin and singleton pregnancies until 29 weeks' gestation but appear higher in twin pregnancies thereafter.

(c) 2021 The Author(s)
Published by S. Karger AG, Basel

\section{Introduction}

Women with twin pregnancies are twice as likely to develop preeclampsia (PE) compared with singleton pregnancies, but the reason for this is unclear $[1,2]$. PE is associated with dysregulation of angiogenic factors soluble fms-like tyrosine kinase-1 (sFlt-1) and placental growth factor (PlGF), and a high sFlt-1/PIGF ratio is a useful predictor of $\mathrm{PE}$ in singleton pregnancies [3-8]. Higher sFlt- 1 concentrations are observed in twin versus singleton pregnancies, suggesting angiogenic factor imbalance may play a role in PE development [9-14].

The Elecsys ${ }^{\circledR}$ sFlt-1/PlGF immunoassay ratio is Certificat de Conformité Européen (In Vitro Diagnostics) approved as a diagnostic and short-term predictive aid for PE $[7,15,16]$. The Prediction of Short-Term Outcome in Pregnant Women with Suspected PE Study (PROGNOSIS) derived and validated an sFlt-1/PIGF ratio cutoff of 38 to predict $\mathrm{PE}$ development. The negative predictive value (NPV) of an sFlt-1/PlGF ratio $\leq 38$ to rule out $\mathrm{PE}$ within 1 week in singleton pregnancies was $99.3 \%$ (95\% confidence interval [CI], 97.9-99.9); the positive predictive value (PPV) to rule in PE within 4 weeks was $36.7 \%$ (95\% CI, 28.4-45.7) $[17,18]$. The sFlt-1/PlGF ratio cutoff of 38 for short-term prediction of PE was also validated in 764 Asian women with singleton pregnancies and suspected PE (PROGNOSIS Asia); an sFlt-1/PlGF ratio of $\leq 38$ had a NPV of $98.6 \%$ (95\% CI, 97.2-99.4) for ruling out PE within 1 week [19]. The clinical utility of the sFlt1/PlGF ratio for ruling out PE was confirmed in the randomized, interventional INSPIRE study on prediction of $\mathrm{PE} /$ eclampsia in women with suspected PE [20].

It is unclear how these data translate to twin pregnancies, as reference ranges for the sFlt-1/PlGF ratio in twin pregnancies have not been published. Maternal sFlt-1
Table 1. Baseline characteristics for women with normal twin pregnancies

\begin{tabular}{lc}
\hline Characteristic & $\begin{array}{l}\text { Women with normal twin } \\
\text { pregnancies, } n=269^{*}\end{array}$ \\
\hline Age, years & \\
$n$ & 269 \\
Mean & 34 \\
Missing & 0 \\
BMI, kg/m & \\
$n$ & 266 \\
Mean & 25 \\
Missing & 3 \\
Gestational age at delivery, weeks & \\
$n$ & 268 \\
Mean & 36 \\
Missing & 1 \\
Mode of delivery, $n(\%)$ & \\
Cesarean section & $156(58)$ \\
Vaginal & $109(41)$ \\
Missing & $4(1)$ \\
\hline
\end{tabular}

Baseline characteristics for the women with singleton pregnancies used as comparisons can be found in the method sheets for the Elecsys sFlt- 1 and Elecsys PlGF immunoassays $[15,16] .{ }^{*}$ PROGNOSIS, $n=22$; STEPS, $n=222$; Case-control, $n=25$.

and PlGF are predominantly produced in the placenta, and the placental mass in twin versus singleton pregnancies differs substantially [21-23]. Maternal blood volume is increased in twin pregnancies, which could also influence the sFlt-1/PlGF ratio measurement [24]. We aimed to establish reference ranges for the Elecsys sFlt-1/PlGF immunoassay ratio in twin pregnancies and assess the short-term PE predictive performance of the sFlt-1/PlGF ratio cutoff of 38 in women with twin pregnancies and suspected PE.

\section{Methods}

Included Studies

Combined data for the sFlt-1/PlGF ratio, sFlt-1, and PlGF concentrations were analyzed from 3 studies in which pregnant women were enrolled: Prediction of Short-Term Outcome in Pregnant Women with Suspected PE Study (PROGNOSIS); the Study of Early-onset PE in Spain (STEPS); and a case-control study of the Elecsys sFlt-1/PlGF immunoassay ratio. The full methodologies for all 3 studies have been previously published [5-7, 17, 18, 25]. PROGNOSIS was a prospective, observational study in pregnant women ( $\geq 18$ years of age; 24 weeks +0 days to 36 weeks +6 days' gestation at the time of the first visit) with suspected PE from 14 countries, which derived and validated an optimal sFlt-1/PlGF ra- 
Table 2. Median, 5th, and 95th percentile values for the Elecsys sFlt-1/PlGF immunoassay ratio in women with normal twin and singleton pregnancies

\begin{tabular}{|c|c|c|c|c|c|c|c|}
\hline & \multicolumn{7}{|c|}{ Gestational age (weeks + days) } \\
\hline & $10+0-14+6$ & $15+0-19+6$ & $20+0-23+6$ & $24+0-28+6$ & $29+0-33+6$ & $34+0-36+6$ & $37+0$-delivery \\
\hline 5th Percentile & 9.59 & 2.33 & 1.95 & 1.33 & 1.49 & 9.80 & 9.69 \\
\hline Median & 21.6 & 6.69 & 4.92 & 3.88 & 10.2 & 38.7 & 38.7 \\
\hline 95th Percentile & 39.1 & 15.6 & 12.5 & 19.0 & 69.2 & 165 & 176 \\
\hline Median & 24.8 & 10.5 & 4.92 & 3.06 & 3.75 & 9.03 & 19.6 \\
\hline 95th Percentile & 54.6 & 25.7 & 14.6 & 10.0 & 33.9 & 66.4 & 112 \\
\hline
\end{tabular}

Participants could provide a sample for $>1$ gestational age window. PlGF, placental growth factor; sFlt-1, soluble fms-like tyrosine kinase-1.

tio cutoff for the short-term prediction of PE [17, 18]. STEPS was a prospective, Spanish study across 10 sites, which evaluated the sFlt-1/PIGF ratio as a predictive marker for early-onset PE in pregnant women aged $\geq 18$ years and at risk of this condition [25]. The prospective, multicenter, case-control study of the Elecsys sFlt-1/PlGF immunoassay ratio enrolled women with normal pregnancy outcome and PE and aimed to establish a gestational phase-adapted sFlt-1/PIGF ratio cutoff to aid the diagnosis of PE [5-7].

\section{Participants}

The reference range analysis included women with twin pregnancies without PE, eclampsia, intrauterine growth restriction, or hemolysis, elevated liver enzymes and low platelet count (HELLP) syndrome. These pregnancies were defined as normal pregnancies for the purposes of this study and will be referred to as such throughout the rest of this manuscript. Twin pregnancies with preterm deliveries were eligible for inclusion in this analysis. Any pregnancies with major fetal malformations or chromosomal disorders were excluded. The sFlt-1/PlGF ratios, sFlt-1, and PlGF concentrations in normal singleton pregnancies, which were used as comparators in this analysis, were obtained from the prospective, multicenter, case-control study of the Elecsys sFlt-1/PlGF immunoassay ratio.

\section{Statistical Analyses}

The median (50th percentile), 5th, and 95th percentile values for the sFlt-1/PlGF ratio, sFlt-1, and PlGF concentrations in normal twin pregnancies were generated for 7 gestational age windows and compared with those in normal singleton pregnancies. The gestational age windows were 10 weeks +0 days to 14 weeks +6 days; 15 weeks +0 days to 19 weeks +6 days; 20 weeks +0 days to 23 weeks +6 days; 24 weeks +0 days to 28 weeks +6 days; 29 weeks +0 days to 33 weeks +6 days; 34 weeks +0 days to 36 weeks +6 days; and 37 weeks +0 days to delivery $[15,16]$. However, it should be noted that Breathnach et al. [26] proposed delivery before 37 weeks in twin pregnancies, and in the largest study to estimate the best moment for pregnancy termination ( $n=29,685$ dichorionic; $n=5,486$ monochorionic pregnancies), Cheong-See et al. [27] suggested that, to minimize perinatal deaths, delivery should be considered at 37 weeks' gestation in uncomplicated dichorionic twin pregnancies and at 36 weeks in monochorionic pregnancies; the same recommendation is given by the National Institute of Health and Care Excellence [28]. If a patient had $>1$ visit in 1 of the specified time frames, the first visit was used. Percentiles were calculated using inverse cumulative distribution function with averaging at discontinuities.

An exploratory analysis was also conducted in women with twin pregnancies and suspected PE (who had or had not gone on to develop PE during their pregnancy) enrolled into the PROGNOSIS study to assess the short-term PE predictive performance of using a cutoff of 38 for the sFlt-1/PlGF ratio. The sensitivity, specificity, PPV, NPV, and area under the receiver operating characteristic curve for predicting the occurrence of $\mathrm{PE}$ within 1 and 4 weeks, based on a cutoff of 38 for the sFlt-1/PlGF ratio, were calculated. All statistical analyses were performed using SAS software (version 9.4).

\section{Results}

\section{Participants}

A total of 269 women with normal twin pregnancies were included in the reference range analysis (PROGNOSIS, $n=22$; STEPS, $n=222$ and case-control study of the Elecsys sFlt-1/PlGF immunoassay ratio, $n=25)$. The mean age of the women was 34 years, the mean BMI was $25 \mathrm{~kg} / \mathrm{m}^{2}$, and the mean gestational age at delivery was 36 weeks. More women in this analysis underwent cesarean section (58\%) versus vaginal delivery (41\%) (Table 1).

A total of 62 women with twin pregnancies and suspected PE from the PROGNOSIS study were included in the exploratory prediction performance analysis, of whom 21 (34\%) developed PE. There were 19 women 
Table 3. Median, 5th, and 95th percentile values for the Elecsys sFlt-1 and Elecsys PlGF immunoassays in women with normal twin and singleton pregnancies

Gestational age (weeks + days)

$10+0-14+6 \quad 15+0-19+6 \quad 20+0-23+6 \quad 24+0-28+6 \quad 29+0-33+6 \quad 34+0-36+6 \quad 37+0-$ Delivery

sFlt-1,pg/mL

Women with twin pregnancies, $n$ 5th Percentile

Median 95th Percentile

Women with singleton pregnancies, $n^{7}$

1,549

1,873

5th Percentile

Median

95th Percentile

PlGF, pg/mL

Women with twin pregnancies, $n$

5th Percentile

Median

95th Percentile

Women with singleton pregnancies, $n^{7}$

5th Percentile

Median

95th Percentile
5
524

$\begin{array}{rr}71 & 208 \\ 1,373 & 1,269 \\ 2,461 & 2,600 \\ 5,278 & 5,144 \\ 157 & 217 \\ 708 & 572 \\ 1,355 & 1,299 \\ 2,807 & 2,997\end{array}$

335

1,316

2,729

6,103

346

618

1,355

3,205

2,501

5
37.9
50.7
93.1
246
28.8
52.6
122

$\begin{array}{rr}71 & 208 \\ 166 & 231 \\ 376 & 455 \\ 1,017 & 1,264 \\ 157 & 217 \\ 66.2 & 119 \\ 135 & 264 \\ 289 & 605\end{array}$

335

218

657

1,727

346

169

465

1,117

65
514
4,448
10,073
319
773
1,742
5,165

$\begin{array}{rr}60 & 49 \\ 624 & 519 \\ 8,408 & 7,279 \\ 29,009 & 17,602 \\ 224 & 176 \\ 992 & 1,533 \\ 2,552 & 3,485 \\ 7,363 & 9,184\end{array}$

65
98.3
495
2,919
319
114
471
1,297

60

20.1

260

3,919

224

78.0

284

984
49
17.4
1,804
6,545
176
54.4
191
862

Participants could provide a sample for $>1$ gestational age window. PlGF, placental growth factor; sFlt-1, soluble fms-like tyrosine kinase-1.

with intrauterine growth restriction who were excluded from the reference range analysis, but who were included in the exploratory performance analysis.

\section{sFlt-1/PIGF Ratio Reference Ranges}

Median, 5th, and 95th percentile values for the sFlt-1/ PlGF ratio in normal twin pregnancies were generated for the 7 gestational age windows assessed (Table 2). No differences were observed in median sFlt-1/PIGF ratios in twin versus singleton pregnancies up to 28 weeks +6 days' gestation (Table 2; Fig. 1). From 29 weeks' gestation onward, median sFlt-1/PlGF ratios were higher for twin (10.2, 38.7, and 38.7) versus singleton (3.75, 9.03, and 19.6) pregnancies for gestational age windows 29 weeks +0 days to 33 weeks +6 days, 34 weeks +0 days to 36 weeks +6 days, and 37 weeks +0 days to delivery, respectively (Table 2; Fig. 1). However, it should be noted that the sample size for gestational window 37 weeks +0 days to delivery for twin pregnancies was small $(n=$ 49). Consistent with the median values, the 5 th and 95 th percentile values for the sFlt-1/PlGF ratio from 29 weeks' gestation onward were higher for twin versus singleton pregnancies (Table 2). Median, 5th, and 95th percentile values for the sFlt-1/PlGF ratio in twin pregnancies dur-

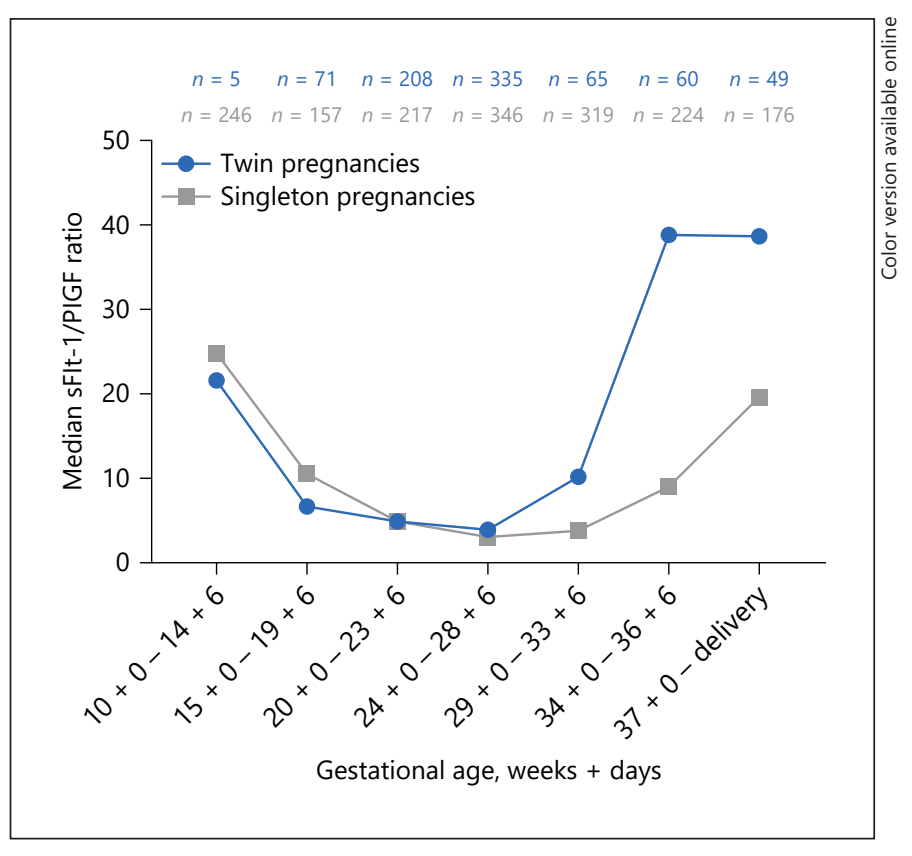

Fig. 1. Comparison of median Elecsys sFlt-1/PlGF immunoassay ratios in normal twin and singleton pregnancies [7]. The lines in the figure link the median values at each gestational age window and are not the result of a model. PlGF, placental growth factor; sFlt-1, soluble fms-like tyrosine kinase-1. 


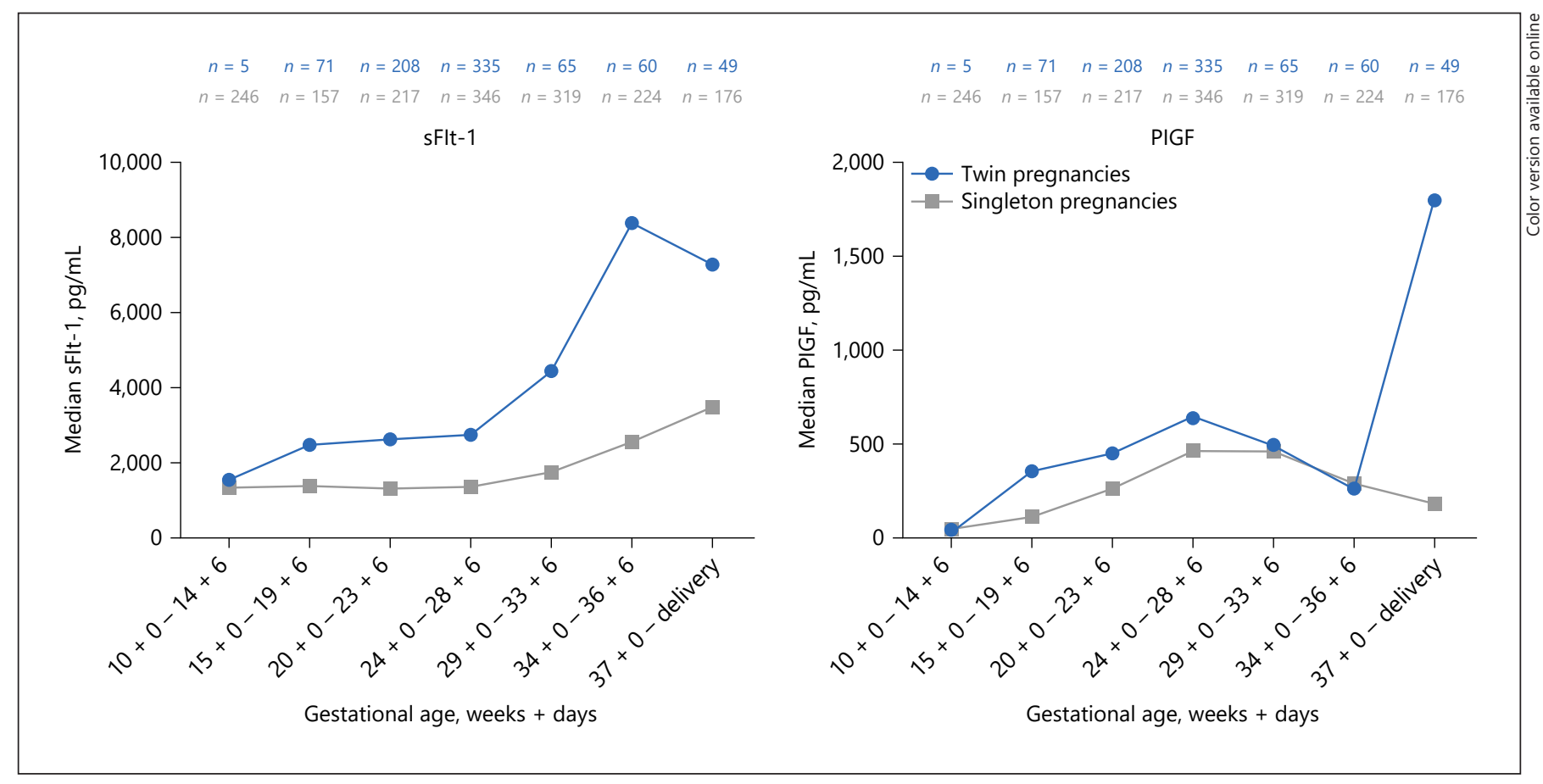

Fig. 2. Comparison of median sFlt-1 and PlGF concentrations in normal twin and singleton pregnancies [7]. The lines in the figure link the median values at each gestational age window and are not the result of a model. PlGF, placental growth factor; sFlt-1, soluble fms-like tyrosine kinase-1.

ing the 2 gestational age windows of 34 weeks +0 days to 36 weeks +6 days $(38.7,9.80$, and 165$)$ and 37 weeks +0 days to delivery $(38.7,9.69$, and 176) were similar (Table 2).

\section{sFlt-1 and PlGF Reference Ranges}

Median, 5th, and 95th percentile values for sFlt- 1 and PlGF concentrations in normal twin pregnancies were also generated for the 7 gestational age windows assessed (Table 3; Fig. 2). Across all gestational age windows, median and 95th percentile sFlt- 1 concentrations were higher in twin versus singleton pregnancies (except for the 95th percentile at 10 weeks +0 days to 14 weeks +6 days' gestation; Table 3; Fig. 2). There was a steeper increase in median and 95th percentile sFlt-1 concentrations after gestational week 29 (Table 3; Fig. 2), which is consistent with the finding that median sFlt-1/PlGF ratios are higher in twin versus singleton pregnancies from 29 weeks' gestation onward (Table 2; Fig. 1). Median and 95th percentile sFlt- 1 concentrations from gestational week 29 onward were around 2-3 times higher in twin versus singleton pregnancies (Table 3; Fig. 2). The 5th percentile sFlt-1 concentrations were higher in twin pregnancies from 15 weeks +0 days' gestation to 28 weeks +6 days' gestation, but lower in twin versus singleton pregnancies from gestational week 29 onwards (Table 3).

Median, 5th, and 95th percentile PIGF concentrations were generally higher in twin versus singleton pregnancies, except at 10 weeks +0 days to 14 weeks +6 days', 29 weeks +0 days to 33 weeks +6 days', and 34 weeks +0 days to 36 weeks +6 days' gestation, when twin and singleton pregnancies showed similar PIGF concentrations (Table 3; Fig. 2). Overall, in women with singleton or twin pregnancies PlGF concentrations increased from 10 weeks +0 days to 14 weeks +6 days' gestation until 28 weeks +6 days' gestation. In women with singleton pregnancies, PlGF values (median, 5th percentile, and 95th percentile) then plateaued before decreasing until delivery. By contrast, in women with twin pregnancies, PlGF values (median and 5 th percentile) decreased until weeks 36 weeks +6 days' gestation before PlGF values (median and 95th percentile) increased sharply from 37 weeks +0 days' gestation until delivery $(260-1,804 \mathrm{pg} / \mathrm{mL}$ [median] and 3,919-6,545 $\mathrm{pg} / \mathrm{mL}$ [95th percentile] at 34 weeks +0 days to 36 weeks +6 days' gestation to 37 weeks +0 days to delivery; Table 3; Figure 2). Again, it should be noted that the sample size for gestational window 37 weeks +0 days to delivery for twin pregnancies was small $(n=49)$. 
Table 4. Exploratory analysis of an sFlt-1/PlGF ratio cutoff of 38 for short-term prediction of $\mathrm{PE}$ in women with twin pregnancies and suspected PE

\begin{tabular}{ll}
\hline PE occurrence & Estimate, \% (95\% CI) \\
\hline Within 1 week & \\
NPV: rule out & $91.9(78.1-98.3)$ \\
PPV: rule in & $20.0(6.8-40.7)$ \\
Sensitivity & $62.5(24.5-91.5)$ \\
Specificity & $63.0(48.7-75.7)$ \\
Within 4 weeks & \\
NPV: rule out & $83.8(68.0-93.8)$ \\
PPV: rule in & $44.0(24.4-65.1)$ \\
Sensitivity & $64.7(38.3-85.8)$ \\
Specificity & $68.9(53.4-81.8)$ \\
\hline
\end{tabular}

NPV, negative predictive value; PE, preeclampsia; PlGF, placental growth factor; PPV, positive predictive value; sFlt-1, soluble fms-like tyrosine kinase-1; CI, confidence interval.

\section{Exploratory Prediction Performance Analysis}

In women with twin pregnancies and suspected $\mathrm{PE}$, an sFlt-1/PlGF ratio $\leq 38$ had an NPV to rule out a diagnosis of PE within 1 week of $91.9 \%$ (95\% CI, 78.1-98.3) and within 4 weeks of $83.8 \%$ (95\% CI, 68.0-93.8; Table 4). The corresponding PPVs of an sFlt-1/PlGF ratio $>38$ to rule in a diagnosis of PE within 1 week was $20.0 \%$ (95\% CI, 6.8-40.7) and within 4 weeks was $44.0 \%$ (95\% CI, $24.4-$ 65.1 ; Table 4). The area under the receiver operating characteristic curve for predicting the occurrence of $\mathrm{PE}$ within 1 week was 0.6 (95\% CI, 0.5-0.8) and within 4 weeks was 0.8 (95\% CI, 0.6-0.9; see online suppl. Fig. 1-4; see www.karger.com/doi/10.1159/000514378 for all online suppl. material).

\section{Chorionicity of Twin Pregnancies}

Although chorionicity data were not recorded in the PROGNOSIS and the Roche case-control study, we evaluated the proportion of monochorionic twin pregnancies in the STEPS study and found 35 monochorionic and 145 dichorionic twin pregnancies in the group of 180 twin pregnancies for which we had chorionicity data. Therefore, the proportion of monochorial to dichorionic twin pregnancies of the evaluable twin pregnancies in the STEPS study was $19.44 \%$ (95\% CI 14.33-25.84). We analyzed the sFlt-1/PlGF ratios between monochorionic and dichorionic pregnancies of the STEPS study and found no statistically significant differences in the sFlt-1/PlGF ratios between monochorionic and dichorionic twin pregnancies across the age windows analyzed (online suppl. Table 1).

\section{Discussion}

Reference ranges have previously been established for the Elecsys sFlt-1/PlGF immunoassay ratio in women with normal singleton pregnancies [7]. The aim of this study was, therefore, to determine reference ranges for the Elecsys sFlt-1/PlGF immunoassay ratio in women with normal twin pregnancies (without PE, eclampsia, intrauterine growth restriction, or HELLP syndrome). Our analysis demonstrated that there were no substantial differences in median, 5th, and 95th percentile values for the sFlt-1/PIGF ratio in women with normal twin pregnancies up to 28 weeks +6 days' gestation versus women with singleton pregnancies. However, from 29 weeks' gestation onward, median, 5th, and 95th percentile values for the sFlt-1/PlGF ratio were higher in women with twin pregnancies, driven primarily by an increase in circulating concentrations of sFlt- 1 . Although placental mass is associated with the production of sFlt1 [21], this does not fully explain why sFlt/PLGF ratios differed after 29 weeks compared to before 29 weeks between single and twin pregnancies, otherwise the differences would also be present at 24-28 weeks; other unknown factors may, therefore, play a role. Interestingly, median, 5th, and 95th percentile values for the sFlt-1/PlGF ratio in twin pregnancies of 29 weeks +0 days to 33 weeks +6 days' gestation $(10.2,1.49$, and 69.2) were similar to the respective values in singleton pregnancies of 34 weeks +0 days to 36 weeks +6 days' gestation $(9.03,1.23$, and 66.4$)$, indicating an earlier increase in the sFlt-1/PlGF ratio in twin pregnancies during the course of pregnancy (Table 2; Fig. 1).

It should be noted that the median PlGF concentration in twin pregnancies of 37 weeks +0 days' gestation to delivery $(1,804 \mathrm{pg} / \mathrm{mL})$ was substantially higher than for the other gestational windows assessed (50.7-657 pg/mL). This may be due to the small sample size for the 37 weeks +0 days to delivery gestational window $(n=49)$. The median sFlt-1/PlGF ratio for twin pregnancies in this gestational window remained higher than in singleton pregnancies.

Our findings are consistent with previously published data, which have shown higher sFlt-1/PIGF ratios after gestational week 28 in normal twin pregnancies than in normal singleton pregnancies [9-11, 13, 14]. For example, 1 study reported a median sFlt-1/PlGF ratio of 13.3 among normal twin pregnancies versus 4.9 among normal singleton pregnancies [10]. Another study reported a median sFlt-1/PlGF ratio of 47.4 in all normotensive women with twin pregnancies, and a median sFlt-1/PlGF ratio of 17.9 in normotensive women with twin pregnan- 
cies before 34 weeks' gestation $[13,14]$. It should be noted that this study used plasma samples to measure the sFlt-1/ PlGF ratio, while the Elecsys sFlt-1 and Elecsys PlGF assays are validated for use with blood serum samples. Another limitation is that the sFlt-1 and PlGF assays of another manufacturer were used in one of these studies, which may differ from the Roche Elecsys immunoassays $[9,29,30]$. However, as the sample sizes were relatively small [13] or provided a limited representation of the full gestational period [9], reference ranges for the sFlt-1/ PIGF ratio could not be established from these previous studies.

Our exploratory prediction performance analysis indicates that using a cutoff of 38 for the sFlt-1/PlGF ratio has an NPV of 91.9 and $83.8 \%$ to rule out PE within 1 and 4 weeks, respectively, in women with twin pregnancies and suspected PE. This is in agreement with the study by Binder et al. [31] which determined that using a cutoff of 38 for the sFlt-1/PlGF ratio has an NPV of 98.8 and $96.4 \%$ to rule out PE within 1 and 2 weeks, respectively. As failure to detect PE can have serious clinical implications, a very high NPV is crucial when assessing patients with suspected PE. For this reason, further analyses are required to determine optimal sFlt-1/PlGF ratio cutoffs for the prediction of PE in women with twin pregnancies, and it remains unclear whether these values should be different to the cutoffs for singleton pregnancies $[7,17,18]$. Until 28 weeks +6 days' gestation, the reference ranges (median, 5th, and 95th percentile values) for the sFlt-1/ PlGF ratio in twin and singleton pregnancies were similar; thus, the cutoffs until this time point should also be similar. However, from 29 weeks + 0 days' gestation onward, median and 95th percentile values for the sFlt-1/ PlGF ratio in women with twin pregnancies were substantially higher than in women with singleton pregnancies. Median sFlt-1/PlGF ratios in normal twin pregnancies of 34 weeks +0 days to 36 weeks +6 days' gestation and 37 weeks +0 days' gestation to delivery were 38.7 and 38.7 , respectively, that is, higher than the validated sFlt-1/ PlGF ratio cutoff of 38 for the short-term prediction of $\mathrm{PE}$ in women with singleton pregnancies and suspected PE $[17,18]$. Therefore, the sFlt-1/PlGF ratio cutoff of 38 for short-term prediction of PE is not useful in twin pregnancies from 29 weeks +0 days' gestation until delivery. This finding is consistent with the results of a small previous study, which showed sFlt-1/PlGF ratios above 38 in some women with twin pregnancies without PE [32].

While we found no statistically significant differences in the sFlt-1/PlGF ratios between monochorionic and dichorionic twin pregnancies, in agreement with Binder et al. [31], Faupel-Badger et al. [9] suggest that monochorionic twin pregnancies have higher values of sFlt-1 and sFlt-1/PlGF ratios. This discrepancy may be due to the low number of cases in the study by Faupel-Badger et al. [9] ( $n=5$ monochorionic pregnancies; $n=36$ dichorionic pregnancies). Although our study has a greater number of cases, it may be underpowered, therefore, an adequate response to this discrepancy would be given by future studies with a larger number of cases.

Our study is the largest to date on the Elecsys sFlt-1/ PlGF immunoassay ratio and angiogenic factors in women with twin pregnancies. The data included are from large, multicenter studies and so are representative of women with twin pregnancies. However, the sample sizes for the gestational age windows after 28 weeks' gestation were smaller, which may limit interpretation of reference ranges for later gestational stages; data from future analyses may provide additional information to more definitively resolve this point. In addition, the amnionicity of the pregnancies was not known in this analysis, and the chorionicity data were only partially available.

In conclusion, this is the first analysis to establish reference ranges for the Elecsys sFlt-1/PlGF immunoassay ratio in women with normal twin pregnancies. Up to 28 weeks +6 days' gestation, median, 5 th, and 95 th percentile values for the sFlt-1/PlGF ratio in twin pregnancies did not differ from values in singleton pregnancies. From 29 weeks of gestation onward, median, 5 th, and 95th percentile values for the sFlt-1/PIGF ratio appear to be higher in twin pregnancies; however, more studies are needed to confirm these findings. These data suggest that the interpretation of the Elecsys sFlt-1/PlGF immunoassay ratio may be useful in women with twin pregnancies. Further studies are needed to determine optimal sFlt-1/PlGF ratio cutoffs for the short-term prediction of PE in women with twin pregnancies.

\section{Acknowledgements}

The authors would like to thank all the women who participated in the included studies, the study site investigators, and the recruitment officers, midwives, and midwifery staff who supported the studies. Third-party medical writing assistance, under the direction of the authors, was provided by Thomas Burton, BMBS (Gardiner-Caldwell Communications, Macclesfield, UK), and was funded by Roche Diagnostics International Ltd., Rotkreuz, Switzerland. ELECSYS is a trademark of Roche. 


\section{Statement of Ethics}

All 3 studies included in this analysis adhered to the Guidelines for Good Clinical Practice, complied with the guidelines for human studies, and were conducted ethically in accordance with the World Medical Association Declaration of Helsinki. Each participating study site provided applicable national/regional Independent Ethics Committee/Institutional Review Board approval of the study protocol (see supplementary material) and associated documents (participant informed consent, participant information) before the start of the clinical part of the study. All women provided written informed consent before enrollment.

\section{Conflict of Interest Statement}

M.d.l.C. reports receiving consulting and lecture fees from Roche Diagnostics. J.L.D. reports consultancy for Roche and Italfarmaco. S.V. reports receiving consulting fees from Roche Diagnostics, Thermo Fisher, and Ferring; lecture fees from Roche Diagnostics, Thermo Fisher, and TEVA; and grant support from Roche Diagnostics and Thermo Fisher. A.I.E. reports receiving speaker fees from Roche Diagnostics and serving on advisory boards for Roche Diagnostics. J.L.B. reports receiving consulting and lecture fees from Roche Diagnostics. J.M.C. reports receiving lecture fees from Roche Diagnostics and Bial. F.C. reports receiving lecture fees and consultancy payment from Roche Diagnostics. I.H. reports receiving speaker fees from Roche Diagnostics and Thermo Fisher and serving on advisory boards for Roche Diagnostics. E.L. reports receiving lecture fees from Cook and receiving lecture fees and serv- ing on advisory boards for Roche Diagnostics. H.K. and M.H. are employed by Roche Diagnostics and hold stock in F. Hoffmann-La Roche Ltd. G.G. and J.S. are employed by Roche Diagnostics. M.H. is an inventor of a pending patent related to sFtl-1/PIGF or endoglin/PIGF ratio to rule out onset of $\mathrm{PE}$ in pregnant women within a certain time period (PCT/EP2013/063115), pending patents related to the dynamic of sFlt-1 or endoglin/PIGF ratio as an indicator for imminent PE and/or HELLP syndrome (PCT/EP2012/072157) and the prediction of postpartum HELLP syndrome, postpartum eclampsia or postpartum PE (PCT/EP2015/051457). A.P. reports receiving lecture fees and serving on advisory boards from Roche Diagnostics, Ferring, EFFIK, Merck and Bial. A.A.d.l.C and J.Á.G-H. report no conflicts of interest. Stefan Verlohren is an Associate Editor of Fetal Diagnosis and Therapy and Elisa Llurba and Editorial Board Member of Fetal Diagnosis and Therapy.

\section{Funding Sources}

This study was supported by funding from Roche Diagnostics International Ltd., Rotkreuz, Switzerland.

\section{Author Contributions}

All the authors contributed to the conception and design of this study and were involved in the interpretation of the data and the development and approval of the manuscript. The data analyses were conducted by H. Kurka and G. Guo.

\section{References}

1 Rao A, Sairam S, Shehata H. Obstetric complications of twin pregnancies. Best Pract Res Clin Obstet Gynaecol. 2004;18(4):557-76.

2 Krotz S, Fajardo J, Ghandi S, Patel A, Keith LG. Hypertensive disease in twin pregnancies: a review. Twin Res. 2002;5(1):8-14.

3 Levine RJ, Maynard SE, Qian C, Lim KH, England LJ, Yu KF, et al. Circulating angiogenic factors and the risk of preeclampsia. $\mathrm{N}$ Engl J Med. 2004;350(7):672-83.

4 Levine RJ, Lam C, Qian C, Yu KF, Maynard SE, Sachs BP, et al. CPEP study group: soluble endoglin and other circulating antiangiogenic factors in preeclampsia. N Engl J Med. 2006; 355:992-1005.

5 Verlohren S, Galindo A, Schlembach D, Zeisler H, Herraiz I, Moertl MG, et al. An automated method for the determination of the sFlt-1/ PIGF ratio in the assessment of preeclampsia. Am J Obstet Gynecol. 2010;202(2):161-e11.

6 Verlohren S, Herraiz I, Lapaire O, Schlembach D, Moertl M, Zeisler H, et al. The sFlt-1/ PIGF ratio in different types of hypertensive pregnancy disorders and its prognostic potential in preeclamptic patients. Am J Obstet Gynecol. 2012;206(1):58-8.

7 Verlohren S, Herraiz I, Lapaire O, Schlembach D, Zeisler H, Calda P, et al. New gestational phase-specific cutoff values for the use of the soluble fms-like tyrosine kinase-1/placental growth factor ratio as a diagnostic test for preeclampsia. Hypertension. 2014;63(2): $346-52$.

8 Maynard SE, Min JY, Merchan J, Lim KH, Li J, Mondal S, et al. Excess placental soluble fms-like tyrosine kinase 1 (sFlt1) may contribute to endothelial dysfunction, hypertension, and proteinuria in preeclampsia. J Clin Invest. 2003;111(5):649-58.

9 Faupel-Badger JM, McElrath TF, Lauria M, Houghton LC, Lim KH, Parry S, et al. Maternal circulating angiogenic factors in twin and singleton pregnancies. Am J Obstet Gynecol. 2015;212(5):636-8.

10 Dröge L, Herraiz I, Zeisler H, Schlembach D, Stepan H, Küssel L, et al. Maternal serum sFlt$1 / \mathrm{PlGF}$ ratio in twin pregnancies with and without pre-eclampsia in comparison with singleton pregnancies. Ultrasound Obstet Gynecol. 2015;45(3):286-93.

11 Maynard SE, Moore Simas TA, Solitro MJ, Rajan A, Crawford S, Soderland P, et al. Circulating angiogenic factors in singleton vs multiple-gestation pregnancies. Am J Obstet Gynecol. 2008;198(2):200-7.

12 Maynard SE, Crawford SL, Bathgate S, Yan J, Robidoux L, Moore M, et al. Gestational angiogenic biomarker patterns in high risk pre- eclampsia groups. Am J Obstet Gynecol. 2013;209(1):53-9.

13 Rana S, Hacker MR, Modest AM, Salahuddin S, Lim KH, Verlohren S, et al. Circulating angiogenic factors and risk of adverse maternal and perinatal outcomes in twin pregnancies with suspected preeclampsia. Hypertension. 2012;60(2):451-8.

14 Rana S, Powe CE, Salahuddin S, Verlohren S, Perschel FH, Levine RJ, et al. Angiogenic factors and the risk of adverse outcomes in women with suspected preeclampsia. Circulation. 2012;125(7):911-9.

15 Roche Diagnostics. Elecsys ${ }^{\curvearrowleft}$ sFlt-1 method sheet. 2020

16 Roche Diagnostics. Elecsys ${ }^{\varpi}$ PlGF method sheet. 2020 .

17 Zeisler H, Llurba E, Chantraine F, Vatish M, Staff AC, Sennström M, et al. Predictive value of the sFlt-1:PlGF ratio in women with suspected preeclampsia. N Engl J Med. 2016; 374(1):13-22.

18 Hund M, Allegranza D, Schoedl M, Dilba P, Verhagen-Kamerbeek W, Stepan H. Multicenter prospective clinical study to evaluate the prediction of short-term outcome in pregnant women with suspected preeclampsia (PROGNOSIS): study protocol. BMC Pregnancy Childbirth. 2014;14:324. 
19 Bian X, Biswas A, Huang X, Lee KJ, Li TK, Masuyama H, et al. Short-term prediction of adverse outcomes using the sFlt-1 (soluble fms-like tyrosine kinase 1)/PlGF (placental growth factor) ratio in Asian women with suspected preeclampsia. Hypertension. 2019; 74(1):164-72.

20 Cerdeira AS, O'Sullivan J, Ohuma EO, Harrington D, Szafranski P, Black R, et al. Randomized interventional study on prediction of preeclampsia/eclampsia in women with suspected preeclampsia: INSPIRE. Hypertension. 2019;74(4):983-90.

21 Bdolah Y, Lam C, Rajakumar A, Shivalingappa V, Mutter W, Sachs BP, et al. Twin pregnancy and the risk of preeclampsia: bigger placenta or relative ischemia? Am J Obstet Gynecol. 2008;198(4):428-6.

22 Cerdeira AS, Kandzija N, Pargmae P, Cooke W, James T, Redman C, et al. Circulating soluble fms-like tyrosine kinase- 1 is placentally derived in normal pregnancy: First in vivo evidence. Pregnancy Hypertens. 2019;16: $145-7$
23 Chau K, Hennessy A, Makris A. Placental growth factor and pre-eclampsia. J Hum Hypertens. 2017;31(12):782-6.

24 Hytten F. Blood volume changes in normal pregnancy. Clin Haematol. 1985;14(3):60112.

25 Perales A, Delgado JL, De La Calle M, GarcíaHernández JA, Escudero AI, Campillos JM, et al. STEPS investigators: sFlt-1/PlGF for earlyonset pre-eclampsia prediction: STEPS (study of early pre-eclampsia in Spain). Ultrasound Obstet Gynecol. 2016;50:373-82.

26 Breathnach FM, McAuliffe FM, Geary M, Daly S, Higgins JR, Dornan J, et al. Optimum timing for planned delivery of uncomplicated monochorionic and dichorionic twin pregnancies. Obstet Gynecol. 2012;119(1):50-9.

27 Cheong-See F, Schuit E, Arroyo-Manzano D, Khalil A, Barrett J, Joseph KS, et al. Prospective risk of stillbirth and neonatal complications in twin pregnancies: systematic review and meta-analysis. BMJ. 2016;354:i4353.

28 National Institute of Health and Care Excellence (NICE): Twin and triplet pregnancy: NICE guideline [NG137]. Available at: https: //www.nice.org.uk/guidance/ng137/chapter/ Recommendations\#timing-of-birth. Accessed 2020 Jun 26
29 Stepan H, Hund M, Dilba P, Sillman J, Schlembach D. Elecsys ${ }^{\circledast}$ and Kryptor immunoassays for the measurement of sFlt- 1 and PlGF to aid preeclampsia diagnosis: are they comparable? Clin Chem Lab Med. 2019;57: 1339-48.

30 Lefèvre G, Hertig A, Guibourdenche J, Lévy P, Bailleul S, Drouin D, et al. Decision-making based on sFlt-1/PlGF ratios: are immunoassay results interchangeable for diagnosis or prognosis of preeclampsia? Clin Chem Lab Med. 2020.

31 Binder J, Palmrich P, Pateisky P, Kalafat E, Kuessel L, Zeisler H, et al. The prognostic value of angiogenic markers in twin pregnancies to predict delivery due to maternal complications of preeclampsia. Hypertension. 2020. 76(1):176-83.

32 Saleh L, Tahitu SIM, Danser AHJ, van den Meiracker AH, Visser W. The predictive value of the sFlt-1/PlGF ratio on short-term absence of preeclampsia and maternal and fetal or neonatal complications in twin pregnancies. Pregnancy Hypertens. 2018;14:222-7. 